\title{
Evaluation of 4D Vascular Flow and Tissue Perfusion in Cerebral Arteriovenous Malformations: Influence of Spetzler-Martin Grade, Clinical Presentation, and AVM Risk Factors
}

\author{
C. Wu, S.A. Ansari, A.R. Honarmand, P. Vakil, M.C. Hurley, B.R. Bendok, J. Carr, T.J. Carroll, and M. Markl
}

\begin{abstract}
BACKGROUND AND PURPOSE: The role of intracranial hemodynamics in the pathophysiology and risk stratification of brain AVMs remains poorly understood. The purpose of this study was to assess the influence of Spetzler-Martin grade, clinical history, and risk factors on vascular flow and tissue perfusion in cerebral AVMs.
\end{abstract}

MATERIALS AND METHODS: 4D flow and perfusion MR imaging was performed in 17 patients with AVMs. Peak velocity and blood flow were quantified in AVM feeding and contralateral arteries, draining veins, and the straight sinus. Regional perfusion ratios (CBF, CBV, and MTT) were calculated between affected and nonaffected hemispheres.

RESULTS: Regarding flow parameters, high-grade AVMs (Spetzler-Martin grade of $>2$ ) demonstrated significantly increased peak velocity and blood flow in the major feeding arteries $(P<.001$ and $P=.004)$ and straight sinus $(P=.003$ and $P=.012)$ and increased venous draining flow $(P=.001)$. The Spetzler-Martin grade significantly correlated with cumulative feeding artery flow $(r=0.85, P<.001)$ and draining vein flow $(r=0.80, P<.001)$. Regarding perfusion parameters, perinidal CBF and CBV ratios were significantly lower $(P<.001)$ compared with the remote ratios and correlated negatively with cumulative feeding artery flow $(r=-0.60, P=.014$ and $r=-0.55, P=.026)$ and draining vein flow $(r=-0.60, P=.013$ and $r=-0.56, P=.025)$. Multiple regression analysis revealed no significant association of AVM flow or perfusion parameters with clinical presentation (rupture and seizure history) and AVM risk factors.

CONCLUSIONS: Macrovascular flow was significantly associated with increasing Spetzler-Martin grade and correlated with perinidal microvascular perfusion in cerebral AVMs. Future longitudinal studies are needed to evaluate the potential of comprehensive cerebral flow and perfusion MR imaging for AVM risk stratification.

ABBREVIATIONS: $\mathrm{c}=$ contralateral hemisphere; $\mathrm{H}=$ hemisphere excluding the nidus; $\mathrm{i}=$ ipsilateral hemisphere; $\mathrm{P}=$ perinidal $\mathrm{PCA}=$ posterior cerebral artery; $\mathrm{R}=$ remote area; SMG = Spetzler-Martin grade

C erebral arteriovenous malformations are associated with an increased risk of intracranial hemorrhage, accounting for $2 \%-4 \%$ of all hemorrhagic strokes annually. ${ }^{1}$ Previous studies have demonstrated the potential risk of hemorrhage following

Received August 5, 2014; accepted after revision December 11.

From the Department of Biomedical Engineering (C.W., T.J.C., M.M.), McCormick School of Engineering, Northwestern University, Evanston, Illinois; and Departments of Radiology (C.W., S.A.A., A.R.H., P.V., M.C.H., B.R.B., J.C., T.J.C., M.M.), Neurological Surgery (S.A.A., M.C.H., B.R.B.), Neurology (S.A.A.), and Otolaryngology (B.R.B.), Feinberg School of Medicine, Northwestern University, Chicago, Illinois. This work was supported by an American Heart Association Predoctoral Fellowship 14PRE18370014, National Institutes of Health-T32-EB005170, National Institutes of Health-R01-HL088437, and Radiological Society of North America Research Seed Grant/Toshiba America Systems RSD1207.

Please address correspondence to Can Wu, MS, Department of Biomedical Engineering and Radiology, Northwestern University, 737 N Michigan Ave, Suite 1600, Chicago, IL 60611; e-mail: canwu2016@u.northwestern.edu

- Indicates open access to non-subscribers at www.ajnr.org

$\nabla$ Indicates article with supplemental on-line videos.

http://dx.doi.org/10.3174/ajnr.A4259
AVM embolization, presumably due to acute alterations in AVM or cerebral hemodynamics. ${ }^{2}$ However, the Spetzler-Martin grade (SMG), widely used for surgical risk stratification in cerebral AVMs, does not provide insight into local or global hemodynamics. ${ }^{3}$ Quantitative assessment of AVM feeding and draining flow, combined with whole-brain tissue perfusion, can potentially characterize both AVM macro- and microvascular pathophysiology. In addition, the relationships between quantitative AVM hemodynamic parameters and the SMG or clinical history (rupture/ seizures) and other risk factors (eg, presence of flow-induced aneurysms or venous stenosis, deep AVM location, and single or deep venous drainage) remain unclear.

In this study, a comprehensive MR imaging and analysis protocol based on 4D flow and DSC perfusion MR imaging was applied for the characterization of 3D AVM macrovascular flow and microvascular brain tissue perfusion. 4D flow MR imaging can measure the 3-directional velocity field with full volumetric coverage of the vessels of interest. ${ }^{4,5}$ Previous studies have shown that 
Demographics and AVM features of the 17 patients with AVMs included in the study

\begin{tabular}{|c|c|c|c|c|c|c|c|}
\hline AVM (No.) & Age (yr) & Sex & Nidal Size $\left(\mathrm{cm}^{3}\right)$ & Drainage & Eloquence & SMG (1-5) & $\begin{array}{c}\text { Major Feeding } \\
\text { Arteries }\end{array}$ \\
\hline 1 & 43 & $M$ & $5.6 \times 4.1 \times 5.1$ & $D+S$ & $\mathrm{~N}$ & 3 & LMCA, LACA, RACA \\
\hline 2 & 29 & M & $4.0 \times 3.5 \times 3.4$ & $D+S$ & $\mathrm{~N}$ & 3 & RPcomA, RPCA \\
\hline 3 & 43 & M & $2.7 \times 3.1 \times 3.1$ & $D+S$ & $Y$ & 3 & RPcomA, RPCA \\
\hline 4 & 21 & $\mathrm{~F}$ & $4.0 \times 3.4 \times 3.8$ & $D+S$ & Y & 4 & LMCA, LPCA, LMMA \\
\hline 5 & 22 & M & $2.5 \times 2.3 \times 2.4$ & $D+S$ & $\mathrm{~N}$ & 2 & RMCA \\
\hline 6 & 68 & $\mathrm{~F}$ & $4.0 \times 3.0 \times 2.5$ & $D+S$ & $\mathrm{Y}$ & 4 & LACA, RACA, RMCA \\
\hline $7^{\mathrm{a}}$ & 34 & M & $3.3 \times 2.3 \times 2.5$ & S & $\mathrm{N}$ & 1 & RACA \\
\hline 8 & 40 & $\mathrm{~F}$ & $3.0 \times 2.0 \times 4.0$ & $D+S$ & $\mathrm{~N}$ & 2 & LMCA, LPCA \\
\hline 9 & 25 & $\mathrm{~F}$ & $2.3 \times 2.3 \times 1.7$ & $D+S$ & $\mathrm{~N}$ & 2 & RMCA \\
\hline 10 & 52 & $\mathrm{~F}$ & $2.4 \times 2.1 \times 2.6$ & S & $\mathrm{N}$ & 1 & LPICA \\
\hline $11^{\mathrm{a}}$ & 16 & $\mathrm{~F}$ & $2.3 \times 2.8 \times 2.8$ & S & Y & 2 & LACA, LMCA \\
\hline 12 & 55 & M & $2.1 \times 3.6 \times 2.6$ & S & $\mathrm{N}$ & 2 & LMCA, LPCA \\
\hline 13 & 49 & M & $1.9 \times 2.4 \times 2.4$ & S & $\mathrm{N}$ & 1 & RMCA \\
\hline 14 & 41 & $\mathrm{~F}$ & $6.6 \times 3.7 \times 5.2$ & $D+S$ & Y & 4 & LMCA, LPCA, LACA \\
\hline 15 & 29 & M & $5.4 \times 3.7 \times 5.5$ & $D+S$ & $\mathrm{Y}$ & 4 & RMCA, RPCA \\
\hline $16^{\mathrm{a}}$ & 36 & M & $3.6 \times 3.4 \times 3.2$ & $\mathrm{~S}$ & $\mathrm{~N}$ & 2 & RACA, RMCA \\
\hline 17 & 66 & M & $2.6 \times 2.3 \times 1.7$ & $D+S$ & $Y$ & 3 & LMCA, LPCA \\
\hline
\end{tabular}

Note:-D indicates deep; S, superficial; L, left; R, right; Y, yes; N, no; LPICA, left posterior inferior cerebellar artery; LACA, left anterior cerebral artery; RACA, right anterior cerebral artery; RPcomA, right posterior communicating artery; LMMA, left middle meningeal artery.

${ }^{a}$ Contralateral arteries not visible in the $4 \mathrm{D}$ flow data.

4D flow MR imaging is highly promising in the evaluation of intracranial hemodynamics in cerebral AVMs. ${ }^{6-8}$ To gain additional information on the impact of AVMs on cerebral tissue perfusion, we used DSC PWI based on a fast spin-echo EPI acquisition during the first pass of contrast agent through the brain vasculature to quantify microvascular perfusion biomarkers. ${ }^{9,10}$ The AVM nidus has relatively low resistance and thus often causes high-flow transnidal shunts, which may result in hypoperfusion in the cerebral parenchyma immediately adjacent to AVMs. However, this so-called "steal effect" with diversion of blood from the adjacent tissue to the AVM nidus is still controversial, and it is unclear whether it should be considered a mechanism for the clinical presentation of cerebral AVMs. ${ }^{11}$

The purpose of this study was to quantitatively evaluate macrovascular flow in AVM arterial and venous networks and microvascular perfusion of the peripheral cerebral parenchyma. We investigated relationships between these quantitative hemodynamic parameters and SMG classification followed by correlation with various AVM symptomatic and anatomic risk factors.

\section{MATERIALS AND METHODS Patient Population}

We studied 17 patients with pretreatment AVMs ( 7 women; mean age, $39 \pm 15$ years) who were identified by institutional neuroradiologists/neurosurgeons and referred for MR imaging between May 2011 and March 2013. Demographics and clinical characteristics of the patients with AVMs are summarized in the Table. The SMG of all AVMs was evaluated on the basis of conventional DSA by experienced neuroradiologists, and ranged from 1 to $4(1, n=$ $3 ; 2, n=6 ; 3, n=4 ; 4, n=4)$. Quantitative analysis of macrovascular flow parameters (peak velocities and mean flow) and microvascular perfusion was performed in all patients with AVMs and was correlated with respect to SMG classification, clinical presentation (history of hemorrhagic events, seizures), and other AVM risk factors (deep AVM location, presence of flow-induced aneurysms or venous stenosis, and single or deep venous drainage). The study was approved by the local institutional review board and was conducted in accordance with Health Insurance Portability and Accountability Act guidelines.

\section{MR Imaging}

All MR imaging measurements were performed on 1.5T (Magnetom Avanto) and 3T (Magnetom Trio) MR imaging scanners (Siemens, Erlangen, Germany) equipped with standard 12-channel head coils. A single dose ( $4 \mathrm{~mL} / \mathrm{s}, 0.1 \mathrm{mmol} / \mathrm{kg}$ bodyweight) of Gd-DTPA contrast agent (Magnevist; Bayer HealthCare Pharmaceuticals, Wayne, New Jersey) was administrated before DSC PWI (spin-echo EPI), followed by a $15-\mathrm{mL}$ saline flush at a rate of 4 $\mathrm{mL} / \mathrm{s}$. Imaging parameters for DSC PWI were as follows: TR/TE $=$ $1100 / 34 \mathrm{~ms}, \mathrm{FOV}=220 \times 220 \mathrm{~mm}^{2}$, spatial resolution $=1.7 \times$ $1.7 \times 5.0 \mathrm{~mm}^{3}$, acquisition time $=2$ minutes and 30 seconds. $4 \mathrm{D}$ flow imaging with $3 \mathrm{D}$ volumetric coverage of the AVM vasculature was applied, and data were acquired with prospective electrocardiogram synchronization. Typical sequence parameters for 4D flow MR imaging were the following: TR/TE $=5.2 / 2.8 \mathrm{~ms}$, flip angle $=15^{\circ}$, velocity sensitivity $=100 \mathrm{~cm} / \mathrm{s}, \mathrm{FOV}=220 \times 160$ $\mathrm{mm}^{2}$, temporal resolution $=42 \mathrm{~ms}$, spatial resolution $=1.1 \times$ $1.1 \times 1.5 \mathrm{~mm}^{3}$, acquisition time $=15-20$ minutes depending on the heart rate of the patient during the scan.

\section{D Flow Data Preprocessing and 3D Blood Flow Visualization}

$4 \mathrm{D}$ flow data were preprocessed by using a customized software programmed in Matlab (MathWorks, Natick, Massachusetts) to filter out background noise and correct for Maxwell terms, eddy current phase offset errors, and velocity aliasing. ${ }^{12}$ In addition, a $3 \mathrm{D}$ phase-contrast MR angiogram was created on the basis of the magnitude and phase difference images. Finally, the preprocessed data were imported into software for 3D blood flow visualization and quantification (EnSight; CEI, Apex, North Carolina). 3D vascular visualization was based on time-resolved 3D pathlines that depict the temporal evolution of blood flow over the cardiac cycle (On-line Videos). Pathlines were emitted from the entire intracranial vasculature provided by the $3 \mathrm{D}$ phase-contrast MR angio- 

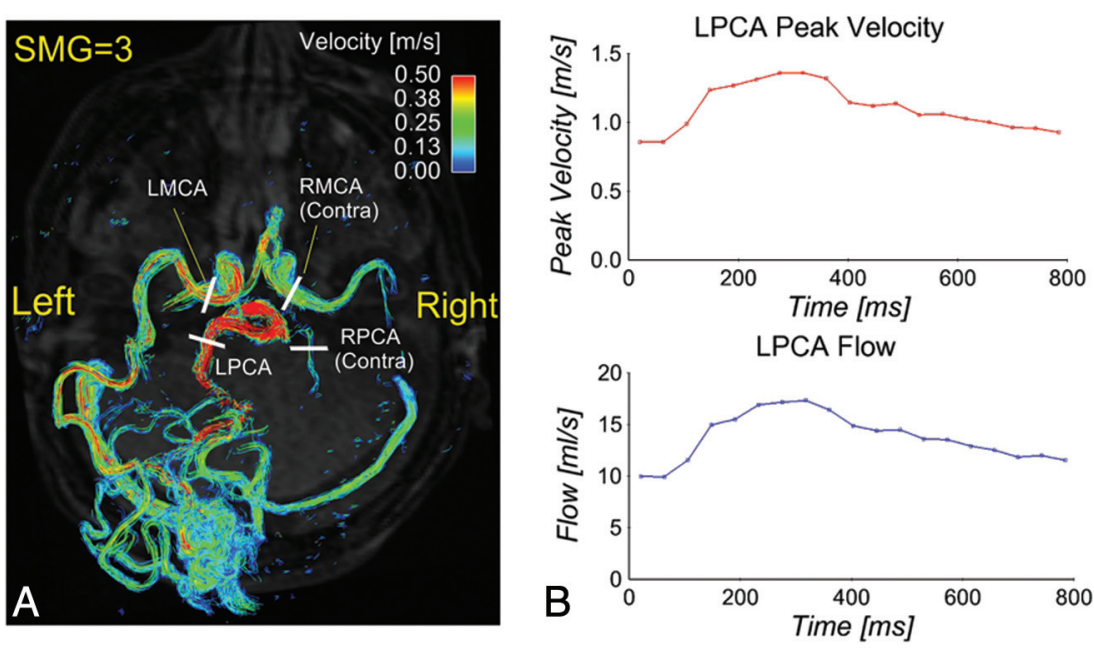

CBF

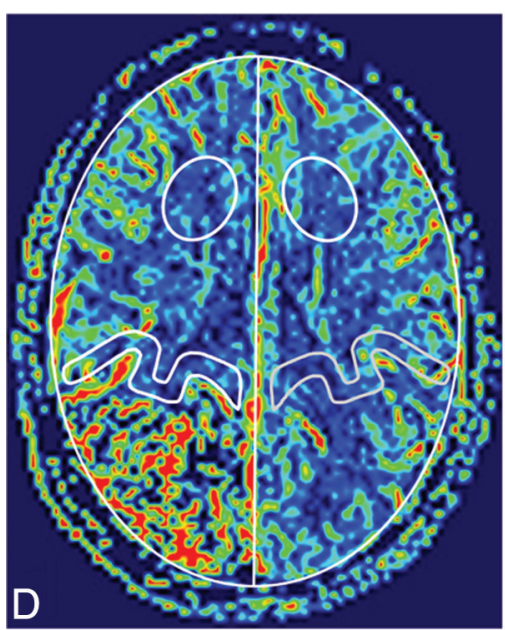

CBV

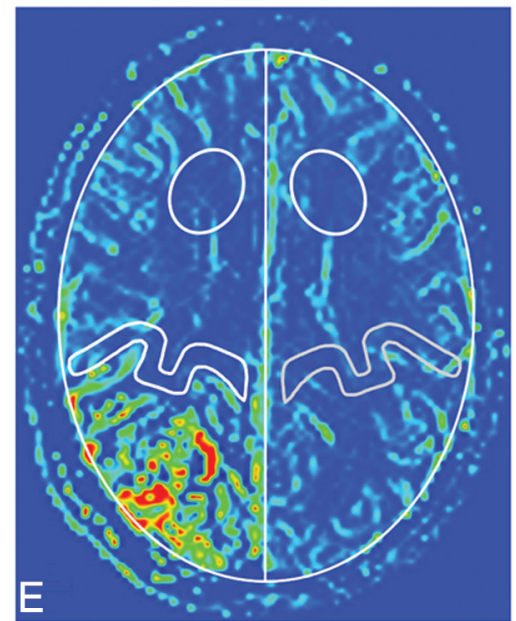

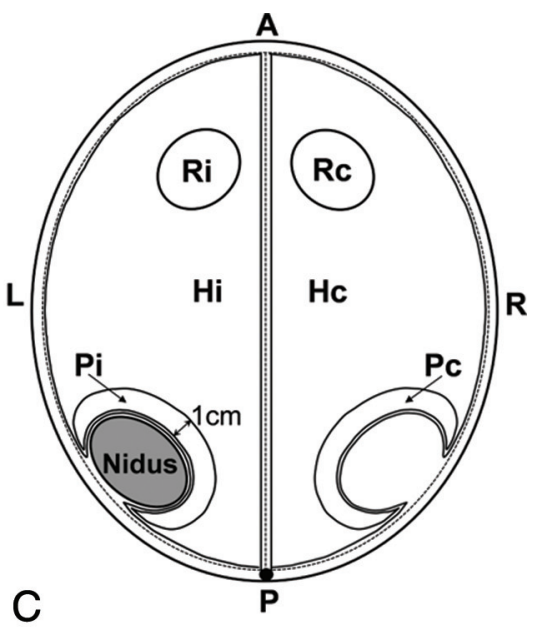

MTT

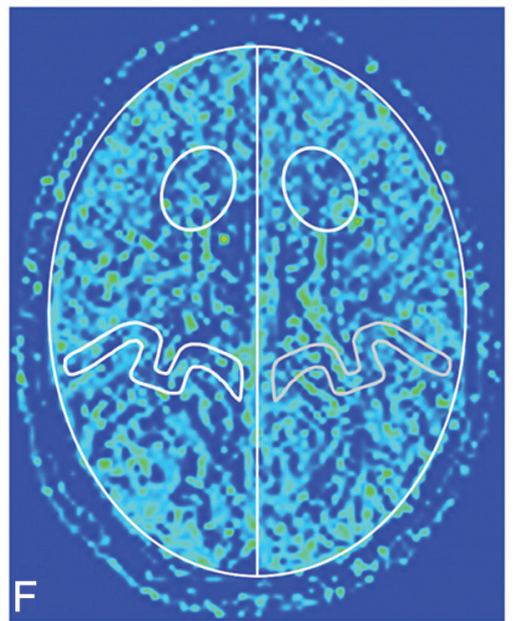

FIG 1. 3D blood flow visualization in a left medial occipital AVM (AVM-17) with time-integrated pathlines (A) and quantification of the peak velocity and blood flow in the major feeding arteries ( $B$, example for $L P C A)$ and a perfusion model (C), including 3 pairs of ROIs ( $\mathrm{Pi} / \mathrm{Pc}, \mathrm{Hi} / \mathrm{Hc}$, and $\mathrm{Ri} / \mathrm{Rc}$ ) used for perfusion analysis in CBF (D), CBV (E), and MTT (F) images (example for AVM-4). LMCA indicates left MCA; LPCA, left PCA; RMCA, right MCA; RPCA, right PCA; Contra, contralateral artery; $\mathrm{Pc}, \mathrm{Hc}$, and Rc, corresponding ROIs in the contralateral hemisphere.

gram data (total virtual particles $=25,000$ ). The color-coding of the time-integrated traces reflects the velocities of blood traveling along the pathlines during 1 cardiac cycle and illustrates the range and magnitude of blood flow velocities within the cardiac cycle (Fig 1A).

\section{Flow Quantification}

For each AVM, 2D analysis planes were manually positioned perpendicularly in all identified feeding and contralateral arteries, draining veins, and the straight sinus by the same observer. The planes were placed consistently across all patients with AVMs (ie, M1 for MCA and P2 for posterior cerebral artery [PCA]) (Fig 1A, example for AVM-17). For each analysis plane, peak velocity (meter/second) and time-averaged blood flow (milliliter/second) over the cardiac cycle were quantified (Fig $1 B$, for the left PCA).

\section{Tissue Perfusion Quantification}

Perfusion maps (CBF, CBV, and MTT) were calculated on-line by using a method described previously, including automatic deter- mination of the arterial input function and deconvolution with the concentration-time curve based on singular value decomposition. ${ }^{9,13}$ Similar to the model proposed by Guo et al, ${ }^{14} 3$ pairs of ROIs at the ipsilateral (i) and contralateral (c) hemispheres were defined as follows (Fig 1C): perinidal (P) area for the detection of potential AVM steal $(\mathrm{Pi} / \mathrm{Pc})$, hemisphere $(\mathrm{H})$ excluding the nidus for global perfusion evaluation $(\mathrm{Hi} / \mathrm{Hc})$, and remote area $(\mathrm{R})$ as an internal control (Ri/Rc). The ROIs were manually delineated by 2 independent observers on a selected CBF map including the largest AVM nidal dimension. ROIs were drawn ipsilateral to the AVM nidus without inclusion of visible vascular territories, and paired contralateral ROIs were automatically created (Fig $1 D-F$, example for AVM-4). Perinidal ROIs were defined consistently for all patients (ie, $1-\mathrm{cm}$ perimeter adjacent to the nidus). The mean values of the ipsilateral-to-contralateral perfusion ratios from the 2 observations were used for subsequent perfusion analysis. ROI selection and calculation of the perfusion parameters were implemented by using an in-house program in Matlab. 


\section{Statistical Analysis}

Flow parameters were compared between AVM feeding and contralateral arteries (2-tailed paired $t$ test). Flow parameters in the major feeding arteries, draining veins, and straight sinus were compared between the low-grade (SMG-A: SMG = 1 and 2) and high-grade (SMG-B: SMG $=3$ and 4) AVM groups (2-tailed $t$ test). The intraclass correlation coefficient $(0.00-0.20$, poor; $0.21-0.40$, fair; $0.41-0.60$, moderate; $0.61-0.80$, good; and $0.81-$ 1.00 , excellent) was used to evaluate the interobserver agreement for perfusion measurements, and perfusion ratios were compared in the 3 ROIs (2-tailed paired $t$ test). In addition, correlation analysis was performed between the SMG and AVM hemodynamics (Spearman correlation), between the AVM nidal volume calculated by $V=\pi / 6 \times a \times b \times c(a, b, c$ are $3 \mathrm{D}$ nidal diameters measured by experienced neuroradiologists on the basis of conventional DSA) ${ }^{15}$ and AVM hemodynamics (Pearson correlation), and between AVM flow parameters and perfusion ratios (Pearson correlation). Multiple regression analysis (stepwise) was performed to identify the potential association of AVM flow or perfusion parameters with clinical presentation and risk factors. All statistical analyses were performed by using the software package SPSS (IBM, Armonk, New York). $P<.05$ was considered statistically significant.

\section{RESULTS}

4D flow and perfusion MR imaging were successfully acquired in all except for 1 patient (AVM-3) whose perfusion acquisition failed due to a technical error (no injection of contrast agent). Patient demographics including SMG and 33 identified feeding arteries are summarized in the Table. Patients with high-grade AVMs (SMG-B, $n=8$ ) had more feeding arteries (at least 2) compared with patients with low-grade AVMs (SMG-A, $n=9$ ). Of the 17 patients in this study, 7 had seizure history; 3 , a previous intracranial hemorrhage; 8 , a deep nidal location; 12 , a flow-induced aneurysm; 3 , a draining venous stenosis; 5 , a single draining vein; and 11, a deep venous drainage. Note that 3 patients (AVM-3, AVM-5, and AVM-7) presented with overt hemorrhage, but they were clinically stable during the scans.

\section{Visualization of AVM Flow and Perfusion}

Figure 2 shows the 3D phase-contrast MR angiogram (top row) and $3 \mathrm{D}$ blood flow visualization (middle row) for 4 representative AVMs with varying SMGs ranging from 1 to 4 . Arterial feeding (solid white arrows) and venous draining (open white arrows) patterns of all AVMs can be clearly appreciated. Perfusion images (Fig 2, bottom row) illustrate corresponding microvascular CBF in the brain capillary bed.

\section{Quantitative Arterial and Venous Flow}

Twenty AVM feeding and corresponding contralateral artery pairs could be identified. Both peak velocities $(1.21 \pm 0.39 \mathrm{~m} / \mathrm{s}$ versus $0.62 \pm 0.23 \mathrm{~m} / \mathrm{s}, P<.001)$ and blood flow $(9.06 \pm 3.05$ $\mathrm{mL} / \mathrm{s}$ versus $2.99 \pm 1.59 \mathrm{~mL} / \mathrm{s}, P<.001)$ in the AVM feeding arteries were significantly higher compared with the contralateral arteries (Fig $3 A,-E$ ). Additionally, major feeding artery peak velocities (Fig 3B) and blood flow (Fig $3 F$ ) for the SMG-B group were significantly increased compared with the SMG-A group
$(1.57 \pm 0.33 \mathrm{~m} / \mathrm{s}$ versus $0.89 \pm 0.26 \mathrm{~m} / \mathrm{s}, P<.001$, and $12.31 \pm$ $2.60 \mathrm{~mL} / \mathrm{s}$ versus $7.68 \pm 2.99 \mathrm{~mL} / \mathrm{s}, P=.004)$, indicating elevated feeding artery flow in high-grade AVMs. For draining veins, the high-grade AVM group demonstrated significantly higher blood flow $(17.54 \pm 8.47 \mathrm{~mL} / \mathrm{s}$ versus $5.24 \pm 1.57 \mathrm{~mL} / \mathrm{s}, P=.001$, Fig $3 G)$. Differences in peak velocities $(0.67 \pm 0.27 \mathrm{~m} / \mathrm{s}$ versus $0.30 \pm$ $0.07 \mathrm{~m} / \mathrm{s}, P=.003)$ and blood flow $(6.34 \pm 4.87 \mathrm{~m} / \mathrm{s}$ versus $1.71 \pm$ $0.37 \mathrm{~m} / \mathrm{s}, P=.012$ ) were also found in the straight sinus (Fig $3 D$, $-H)$.

\section{Microvascular Perfusion Ratios}

Interobserver agreement for the perfusion measurement was good to excellent (CBF: intraclass correlation coefficient $=0.78$; CBV: intraclass correlation coefficient $=0.86$; MTT: intraclass correlation coefficient $=0.76)$. A significantly lower perinidal CBF ratio was identified compared with the remote ratio $(0.85 \pm$ 0.12 versus $0.99 \pm 0.07, P<.001)$ in agreement with the lower perinidal CBV ratio compared with the remote ratio $(0.83 \pm 0.13$ versus $1.00 \pm 0.08, P<.001)$, indicating the presence of perinidal hypoperfusion (AVM steal) (Fig 4A). However, perinidal and remote MTT ratios were not significantly different $(0.98 \pm 0.09$ versus $1.03 \pm 0.10, P=.110$ ). In addition, no significant difference was observed between remote and hemispheric perfusion ratios (CBF: $P=.231$, CBV: $P=.481$, and MTT: $P=.635$ ). Perfusion ratios were also not significantly different between lowand high-grade AVM subgroups (Fig 4B-D).

\section{Relationships among SMG, Nidal Volume, Arterial and Venous Flow, and Perfusion Ratios}

Strong and significant relationships were observed between SMG and maximum peak velocities in the feeding arteries $(r=$ $0.78, P<.001)$, cumulative blood flow in all feeding arteries $(r=0.85, P<.001)$, and blood flow in the major draining vein $(r=0.80, P<.001)$ and in the straight sinus $(r=0.67, P=.003)$. Further analysis revealed significant relationships of AVM nidal volume with cumulative blood flow in all feeding arteries $(r=$ $0.82, P<.001)$ and draining veins $(r=0.66, P=.004)$. Moreover, the perinidal $\mathrm{CBF}$ ratio correlated negatively with cumulative feeding artery flow $(r=-0.60, P=.014)$ and draining vein flow $(r=-0.60, P=.013)$. A similar negative correlation was observed between the perinidal CBV ratio and cumulative flow in the feeding arteries $(r=-0.55, P=.026)$ and draining veins $(r=$ $-0.56, P=.025)$.

\section{Relationship between AVM Hemodynamics and Risk Factors}

Multiple regression analysis revealed no significant association of AVM vascular flow or perfusion parameters with clinical presentation (rupture and seizure history) and AVM risk factors (deep AVM location, presence of flow-induced aneurysms or venous stenosis, and single or deep venous drainage).

\section{DISCUSSION}

The results of this study demonstrate the potential of $4 \mathrm{D}$ flow and DSC perfusion MR imaging for the comprehensive evaluation of macro- and microvascular hemodynamics in cerebral AVMs. 4D flow MR imaging provided 3D visualization of complex AVM 
$S M G=1$
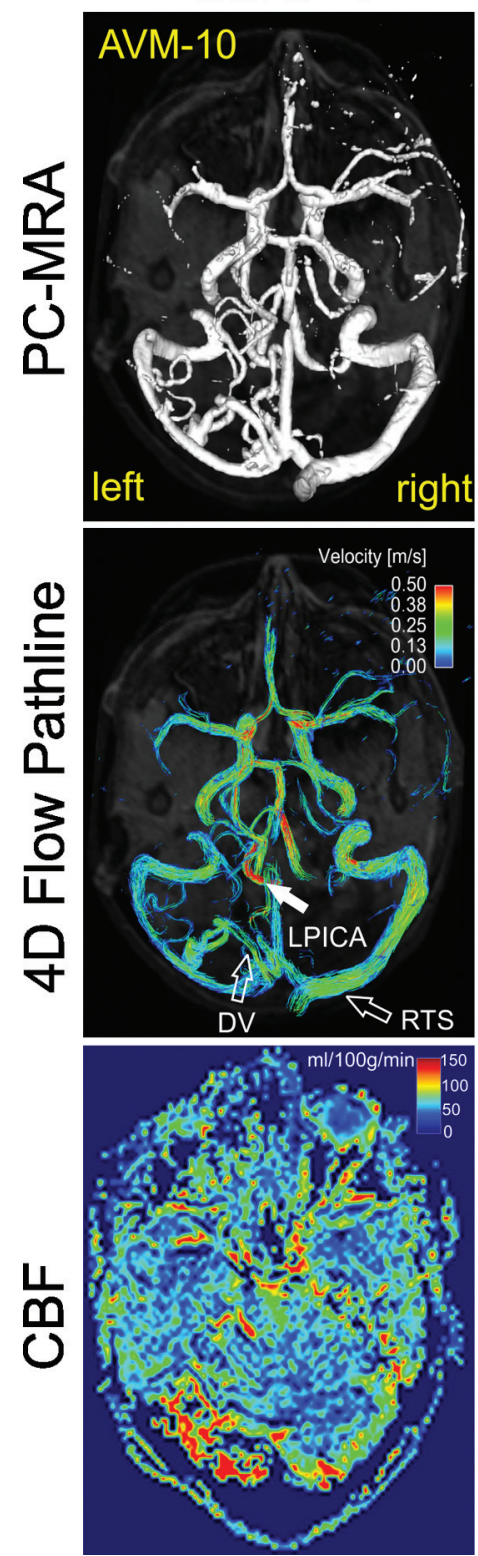

$\mathrm{SMG}=2$
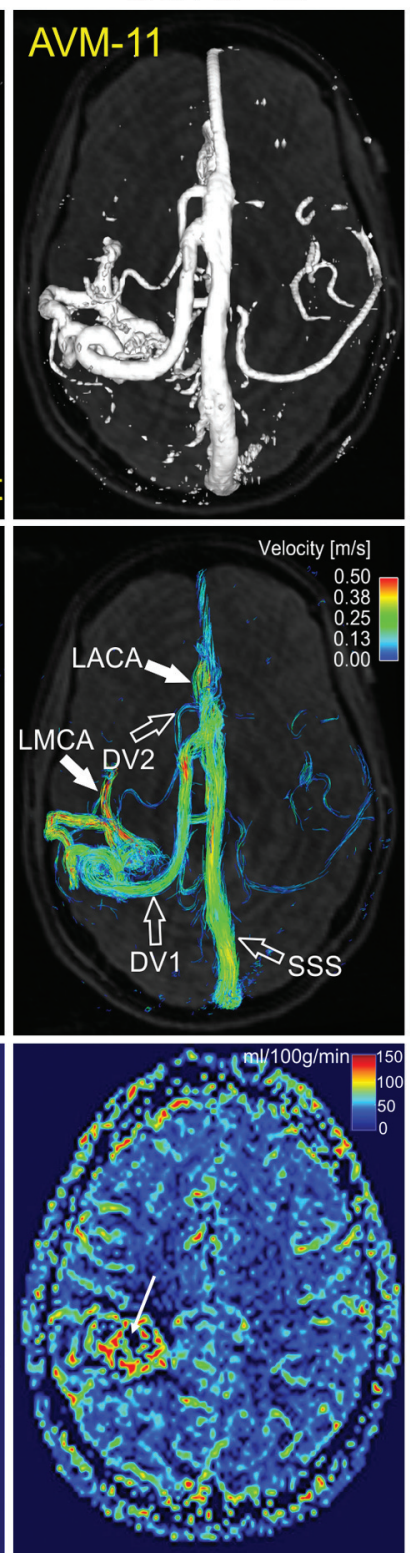

$S M G=3$
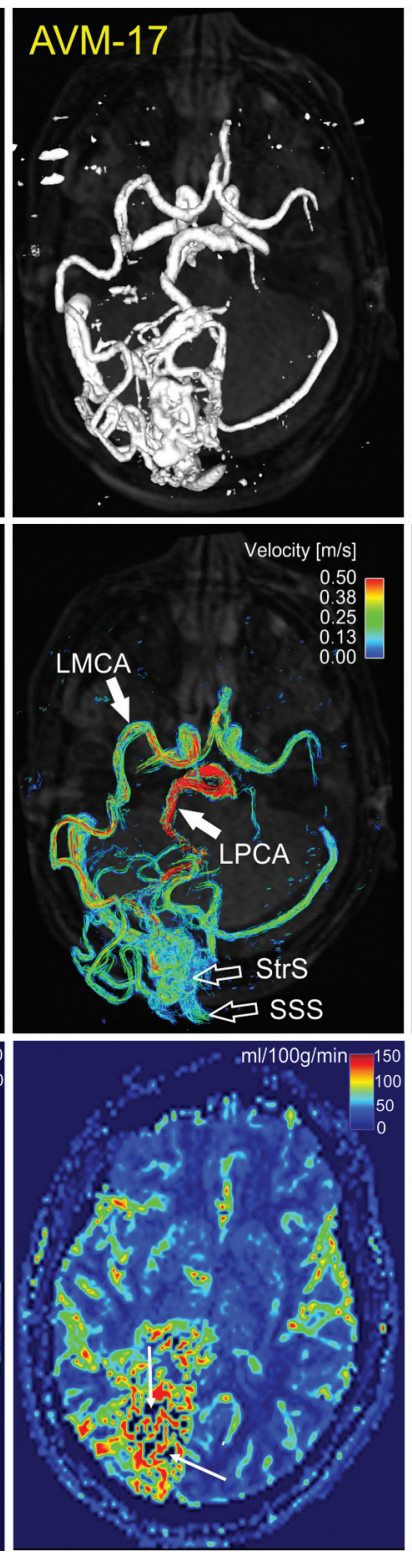

$\mathrm{SMG}=4$
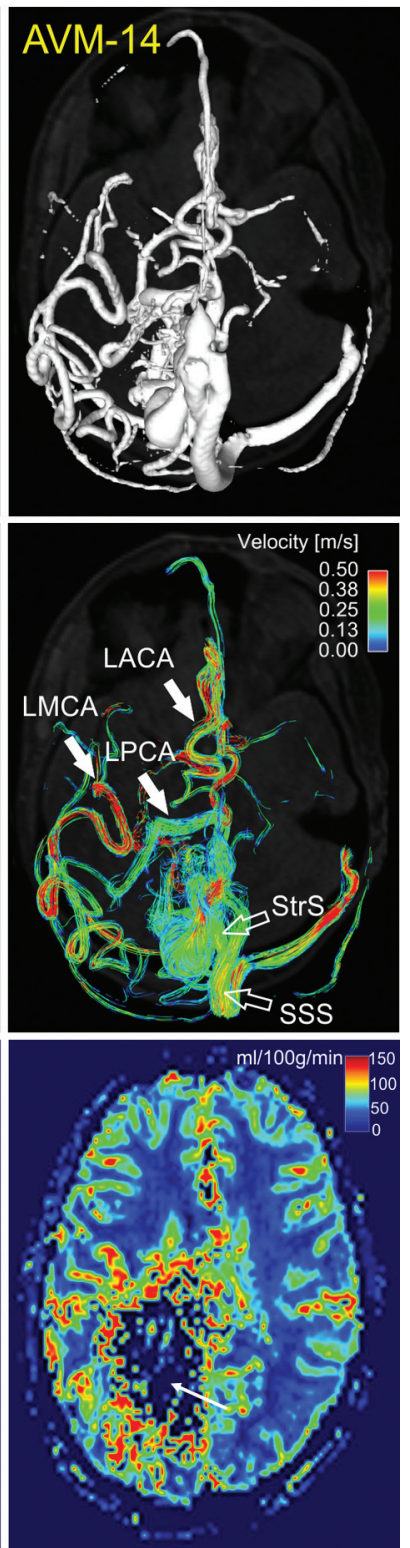

FIG 2. Phase-contrast MRA (PC-MRA), time-integrated 3D pathlines, and perfusion (CBF) for 4 patients with AVMs (SMG = 1-4). AVM arterial feeding (solid white arrows) and venous draining patterns (open white arrows) can be clearly appreciated in the PC-MRA and 4D flow pathlines. The draining veins of AVM-17 and AVM-14 are obscured by pathlines and are not shown. 4D flow pathlines also illustrate the distribution of blood flow velocities in the entire brain. Note the flow voids (thin white arrows) within the nidus for AVM-11, AVM-17, and AVM-14 due to high transnidal shunt flow. LPICA indicates left posterior inferior cerebellar artery; LACA, left anterior cerebral artery; DV, draining vein; RTS, right transverse sinus; StrS, straight sinus; SSS, superior sagittal sinus.

arterial feeding and venous draining patterns and offered quantitative flow characterization of large intracranial AVM vessels. DSC PWI was used to characterize the relative differences in microvascular perfusion parameters. Our findings demonstrate the association of the SMG classification with flow parameters and also reveal significant relationships between macrovascular flow and perinidal tissue perfusion but indicate no significant association of AVM flow or perfusion parameters with symptomatic and anatomic risk factors.

Cerebral AVMs are highly heterogeneous and complex lesions that preclude standardized clinical protocols and uniform paradigms for risk stratification and treatment planning. Initial results from A Randomized trial of Unruptured Brain Arteriovenous malformations (ARUBA) have questioned the benefit-to-risk ratio of interventional therapy versus medical management in unruptured AVMs. ${ }^{16}$ Previous studies indicated that perinidal tissue perfusion and flow dynamics in the AVM feeding and draining systems play an important role in assessing the intra- and postoperative hemorrhagic risk. ${ }^{17,18}$ Hence, patient- and AVM-specific risk stratification with individualized AVM hemodynamic evaluation may be an increasingly important analysis required to demonstrate a benefit of embolization or surgical treatment in highrisk AVMs.

A growing number of AVM risk factors have been demon- 
Feeders/Contra Major Feeders
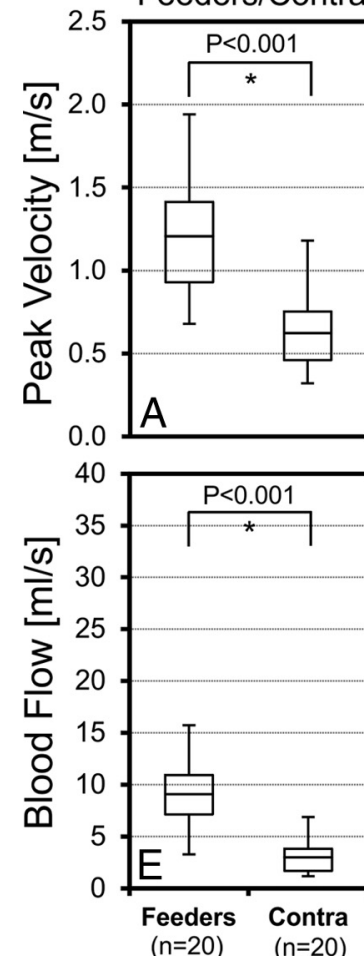

$(n=20) \quad(n=20)$
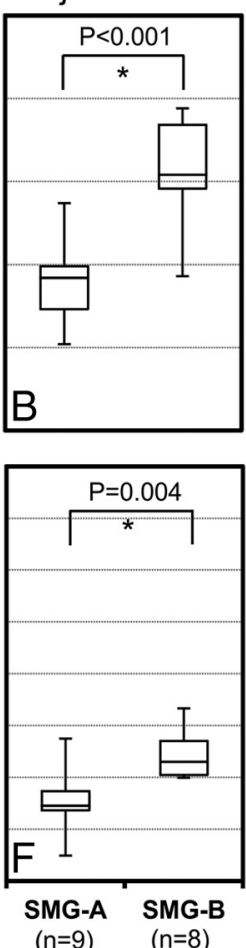

Draining Veins
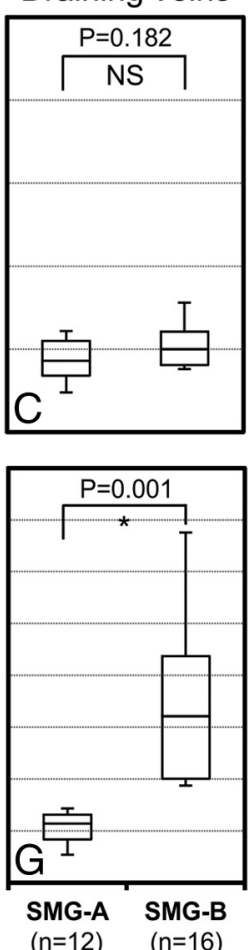

FIG 3. Distribution of peak velocities and blood flow in the AVM feeding (Feeders) and corresponding normal contralateral arteries (Contra) $(A$ and $E$ ), as well as in the major feeding arteries ( $B$ and $F$ ), draining veins ( $C$ and $G$ ), and the straight sinus ( $D$ and $H$ ) for the low (SMG-A) and high (SMG-B) grade AVM groups. The feeding arteries had significantly higher peak velocity and blood flow compared with the normal contralateral arteries. The high-grade AVM group (SMG-B) had significantly higher peak velocity and blood flow in the major feeding arteries and the straight sinus and significantly higher blood flow in the draining veins compared with the low-grade group (SMG-A). The asterisk indicates significant difference with $P<.05 ; \mathrm{NS}$, not significant.
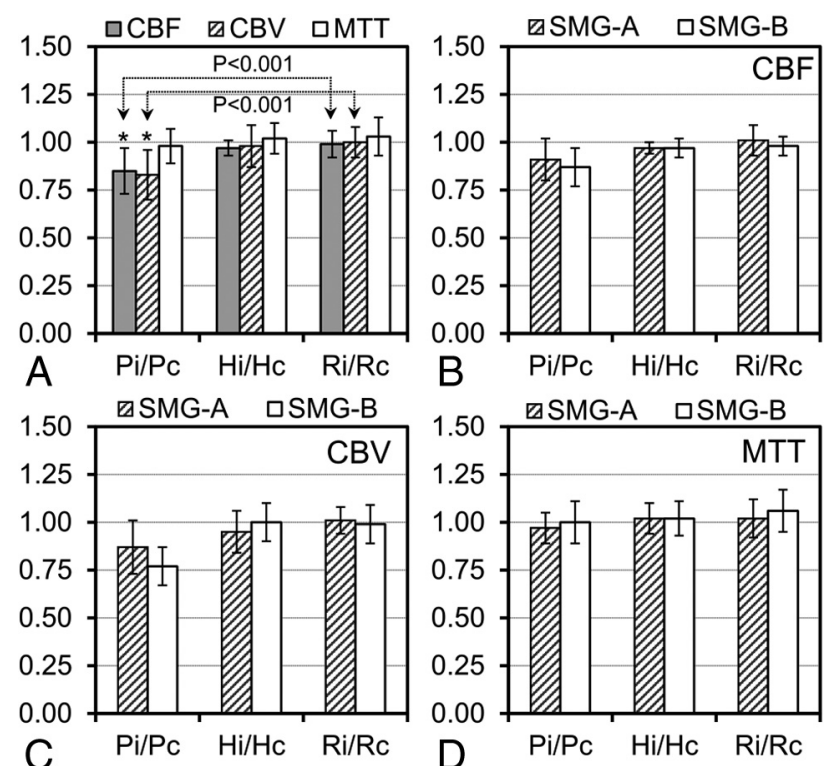

FIG 4. Ipsilateral-to-contralateral perfusion ratios (CBF, CBV, and MTT ratios) in the 3 pairs of ROIs ( $\mathrm{Pi} / \mathrm{Pc}, \mathrm{Hi} / \mathrm{Hc}$, and $\mathrm{Ri} / \mathrm{Rc}$ ) for $16 \mathrm{AVMs}$ $(A)$ and perfusion ratios in the low (SMG-A, $n=9$ ) and high (SMG-B, $n=7$ ) grade AVM groups for $\mathrm{CBF}(B), \mathrm{CBV}(C)$, and MTT $(D)$. The asterisk indicates a significant difference between perinidal ( $\mathrm{Pi} / \mathrm{PC})$ and remote (Ri/Rc) perfusion ratios.

Straight Sinus
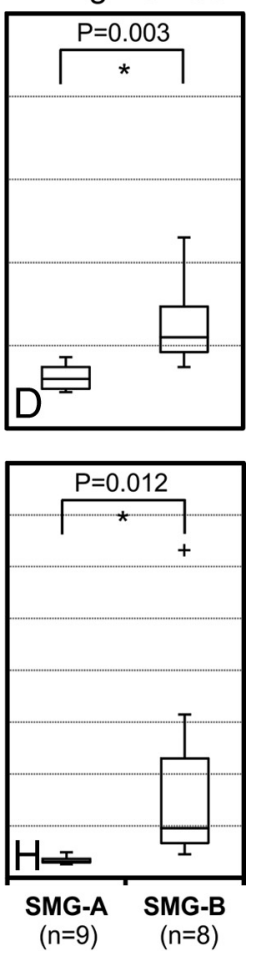

strated to be associated with increased risk of future hemorrhage, such as prior intracranial hemorrhage, ${ }^{1,19}$ a single draining vein, ${ }^{19}$ flow-induced aneurysms, ${ }^{20}$ and deep location. ${ }^{1,21}$ However, the mechanisms of how these factors confer increased hemorrhagic risk remain unclear, with a paucity of data on the role of quantitative AVM hemodynamics. Illies et $\mathrm{al}^{22}$ reported that a previous intracranial hemorrhage was the only risk factor associated with a significant increase of the relative transit time. In our study, we observed no direct connection between AVM hemodynamic parameters and a history of intracranial hemorrhage based on a relatively small sample size. In addition, our findings revealed no significant association of AVM flow or perfusion parameters with seizure history and other anatomic risk factors.

An early 4D flow study by Chang et $\mathrm{al}^{7}$ identified significantly higher time-averaged flow and velocity in the ipsilateral arteries compared with normal contralateral vessels. Similar to their findings, our results demonstrate significantly higher peak velocity and blood flow in the feeding arteries compared with normal contralateral arteries. In addition, our results demonstrate that the SMG directly correlates with arterial flow parameters: The major feeding arteries in high-grade AVMs had significantly higher peak velocities and blood flow in comparison with low-grade AVMs. In addition to SMG, correlations were also identified between AVM nidal volume and cumulative arterial/venous blood flow, indicating that nidal size in the SMG grading system is presumably the dominating factor responsible for different AVM hemodynamics.

A subset of the patients enrolled in this study (AVM 1-14) have been included in a previously reported pilot $4 \mathrm{D}$ flow MR imaging study. ${ }^{6}$ The present study included additional recruited patients, refined flow-quantification analysis, and a combination with DSC perfusion MR imaging that was not described in the previous study. In the previous study, a direct relationship between SMG and increased blood flow velocities was found only for draining veins by visual grading of time-integrated pathlines on a 3 -point scale $(0:<0.25 \mathrm{~m} / \mathrm{s}, 1:<0.5 \mathrm{~m} / \mathrm{s}, 2:>0.5 \mathrm{~m} / \mathrm{s}){ }^{6}$ Reanalysis of these data by using regional flow quantification as used in this study demonstrated the following arterial feeding and venous draining peak velocities in 3 SMG groups: $\mathrm{SMG}=1$ $(0.68 \pm 0.29 \mathrm{~m} / \mathrm{s} ; 0.32 \pm 0.13 \mathrm{~m} / \mathrm{s}), \mathrm{SMG}=2(0.79 \pm 0.23 \mathrm{~m} / \mathrm{s} ;$ $0.38 \pm 0.16 \mathrm{~m} / \mathrm{s})$, and $\mathrm{SMG}>3(0.97 \pm 0.54 \mathrm{~m} / \mathrm{s} ; 0.50 \pm 0.24$ $\mathrm{m} / \mathrm{s})$. In comparison, the previously used visual velocity grading resulted in $\mathrm{SMG}=1(1.7 \pm 0.6 ; 0.6 \pm 0.6), \mathrm{SMG}=2(1.4 \pm 0.5$; $0.8 \pm 0.8)$, and SMG $>3(1.6 \pm 0.5 ; 1.2 \pm 0.6)$. These results 
indicate that visual grading analysis agreed with quantitative measures for venous velocities but resulted in lower arterial velocities in group SMG $=2$ compared with group $\mathrm{SMG}=1$. In contrast, quantitative analysis showed consistently increased arterial velocities with increasing SMG. The discrepancy may be caused by a different magnitude of velocities in the arterial $(0.88 \pm 0.44 \mathrm{~m} / \mathrm{s})$ and venous $(0.43 \pm 0.21 \mathrm{~m} / \mathrm{s})$ systems. The previously used visual grading method may thus not have allowed accurate arterial velocity measurement for arterial velocities of $>0.5 \mathrm{~m} / \mathrm{s}$.

Fast arterial-to-venous blood flow velocities in cerebral AVMs present a great challenge for the quantification of arteriovenous shunting with conventional imaging techniques. It is problematic to quantify arteriovenous shunting simply on the basis of absolute arterial or venous blood flow velocities without consideration of the complex and heterogeneous nidal vascular network. DSA, time-resolved contrast-enhanced MRA, and arterial spin-labeled MR imaging have been shown to provide improved quantification of arteriovenous shunting measured with venous arrival time or arterial-to-venous transit time. ${ }^{15,23,24}$

A previous DSC PWI study demonstrated AVM steal based on significantly higher $\mathrm{Hi} / \mathrm{Hc}$ ratios of $\mathrm{CBF}$ and $\mathrm{CBV}$ in 19 patients with AVMs in comparison with healthy subjects. ${ }^{14}$ Fiehler et $\mathrm{al}^{25}$ reported decreased perfusion ratios between affected and nonaffected hemispheres in the MCA and PCA territories accounting for AVM steal. In our study, there were significantly lower perinidal $\mathrm{CBF}$ and $\mathrm{CBV}$ ratios compared with the remote ratios indicating presence of perinidal hypoperfusion ("AVM steal”). Our perfusion imaging findings were not affected if the 2 ruptured AVMs (AVM-5 and AVM-7) were excluded from the analysis. Flow voids were observed within a high-flow AVM nidus (Fig 2, thin white arrows) due to spin-echo PWI. The signal voids may be alleviated by increasing section thickness and shortening the TR.

A major limitation of this study is the small number of patients with AVMs $(n=17)$, which underlines the relatively rare pathology of cerebral AVMs. Nevertheless, to our knowledge, this is the largest cohort to date for quantitative evaluation of flow parameters in both AVM arterial and venous systems as well as microvascular brain tissue perfusion. Future studies with larger patient cohorts and healthy control groups are warranted to increase the statistical power and further clarify the relevance of macrovascular flow and microvascular tissue perfusion in cerebral AVMs.

Another limitation related to $4 \mathrm{D}$ flow MR imaging is its inability to capture the wide range of velocities in cerebral AVMs (ie, fast feeding artery flow versus slow venous flow). Dual velocity encoding is promising to improve the velocity-to-noise ratio for vascular flow quantification. ${ }^{26}$ In addition, small vessels (eg, feeding arteries and draining veins with diameters of $<3 \mathrm{~mm}$ ) were difficult to visualize and quantify with the current $4 \mathrm{D}$ flow technique due to limited spatial resolution, which may be improved by advanced imaging accelerating techniques to increase spatial resolution while keeping total scan times at acceptable levels. ${ }^{27}$ Moreover, the AVMs included in this study had a different number of feeding and draining vessels, which may bias the statistical results for the peak velocities and blood flow.

The perfusion method used in this study has been successfully applied in the evaluation of brain tumor, ${ }^{10}$ but it was only validated against the reference standard (PET) for gradient-echo
PWI. ${ }^{28}$ In addition, a wide variability in contralateral white matter $\mathrm{CBF}$ values was reported in the presence of vascular pathology. ${ }^{29}$ Thus, we were cautious to use the values in our study as quantitative perfusion measurement in cerebral AVMs without further validation. Instead, the relative perfusion ratios between the affected and nonaffected hemispheres were calculated to characterize the potential perfusion disturbance. Moreover, although a single section with the largest nidal dimension was considered to represent the most significant hemodynamics in cerebral AVMs,${ }^{14} 3 \mathrm{D}$ assessment of brain tissue perfusion would provide better characterization of microvascular hemodynamic disturbance in cerebral AVMs. ${ }^{22}$

\section{CONCLUSIONS}

4D flow and DSC perfusion MR imaging enabled quantitative hemodynamic assessment of cerebral AVMs in both AVM arterial feeding and venous drainage systems as well as the capillary microperfusion of peripheral brain tissue. Our results demonstrate the association of SMG with AVM arterial and venous flow as well as significant relationships between macrovascular flow and changes in cerebral perfusion parameters. Future studies in larger patient cohorts including longitudinal follow-up are needed to evaluate the potential of comprehensive MR imaging for the assessment of both cerebral flow and perfusion for improved AVM risk stratification.

Disclosures: Can Wu-RELATED: Grant: American Heart Association, ${ }^{\star}$ Comments: American Heart Association Predoctoral Fellowship 14PRE18370014. Bernard R. Bendok-RELATED: Grant: MicroVention (research grant). * James Carr-UNRELATED: Consultancy: Guerbet; Grants/Grants Pending: Bayer, ${ }^{*}$ Astellas, ${ }^{*}$ Siemens*; Payment for Lectures (including service on Speakers Bureaus): Siemens. *Money paid to the institution.

\section{REFERENCES}

1. Hernesniemi JA, Dashti R, Juvela S, et al. Natural history of brain arteriovenous malformations: a long-term follow-up study of risk of hemorrhage in 238 patients. Neurosurgery 2008;63:823-29; discussion 829-31

2. Heidenreich JO, Hartlieb S, Stendel R, et al. Bleeding complications after endovascular therapy of cerebral arteriovenous malformations. AJNR Am J Neuroradiol 2006;27:313-16

3. Spetzler RF, Martin NA. A proposed grading system for arteriovenous malformations. J Neurosurg 1986;65:476-83

4. Bammer R, Hope TA, Aksoy M, et al. Time-resolved 3D quantitative flow MRI of the major intracranial vessels: initial experience and comparative evaluation at $1.5 \mathrm{~T}$ and $3.0 \mathrm{~T}$ in combination with parallel imaging. Magn Reson Med 2007;57:127-40

5. Markl M, Frydrychowicz A, Kozerke S, et al. 4D flow MRI. J Magn Reson Imaging 2012;36:1015-36

6. Ansari SA, Schnell S, Carroll T, et al. Intracranial 4D flow MRI: toward individualized assessment of arteriovenous malformation hemodynamics and treatment-induced changes. AJNR Am J Neuroradiol 2013;34:1922-28

7. Chang W, Loecher MW, Wu Y, et al. Hemodynamic changes in patients with arteriovenous malformations assessed using high-resolution $3 \mathrm{D}$ radial phase-contrast MR angiography. AJNR Am J Neuroradiol 2012;33:1565-72

8. Hope MD, Purcell DD, Hope TA, et al. Complete intracranial arterial and venous blood flow evaluation with 4D flow MR imaging. AJNR Am J Neuroradiol 2009;30:362-66

9. Srour JM, Shin W, Shah S, et al. SCALE-PWI: A pulse sequence for absolute quantitative cerebral perfusion imaging. J Cereb Blood Flow Metab 2011;31:1272-82 
10. Carroll TJ, Horowitz S, Shin W, et al. Quantification of cerebral perfusion using the "bookend technique": an evaluation in CNS tumors. Magn Reson Imaging 2008;26:1352-59

11. Mast H, Mohr JP, Osipov A, et al. 'Steal' is an unestablished mechanism for the clinical presentation of cerebral arteriovenous malformations. Stroke 1995;26:1215-20

12. Bock J, Kreher BW, Hennig J, et al. Optimized pre-processing of time-resolved 2D and 3D phase contrast MRI data. In: Proceedings of the 15th Scientific Meeting of the International Society for Magnetic Resonance in Medicine, Berlin, Germany. May 19-25, 2007:3138

13. Carroll TJ, Rowley HA, Haughton VM. Automatic calculation of the arterial input function for cerebral perfusion imaging with MR imaging. Radiology 2003;227:593-600

14. Guo WY, Wu YT, Wu HM, et al. Toward normal perfusion after radiosurgery: perfusion $M R$ imaging with independent component analysis of brain arteriovenous malformations. AJNR Am J Neuroradiol 2004;25:1636-44

15. Todaka T, Hamada J, Kai Y, et al. Analysis of mean transit time of contrast medium in ruptured and unruptured arteriovenous malformations: a digital subtraction angiographic study. Stroke 2003;34:2410-14

16. Mohr JP, Parides MK, Stapf C, et al. Medical management with or without interventional therapy for unruptured brain arteriovenous malformations (ARUBA): a multicentre, non-blinded, randomised trial. Lancet 2014;383:614-21

17. Ogasawara K, Yoshida K, Otawara Y, et al. Cerebral blood flow imaging in arteriovenous malformation complicated by normal perfusion pressure breakthrough. Surg Neurol 2001;56:380-84

18. Young WL, Prohovnik I, Ornstein E, et al. Monitoring of intraoperative cerebral hemodynamics before and after arteriovenous malformation resection. Anesth Analg 1988;67:1011-14

19. Pollock BE, Flickinger JC, Lunsford LD, et al. Factors that predict the bleeding risk of cerebral arteriovenous malformations. Stroke 1996;27:1-6
20. Redekop G, TerBrugge K, Montanera W, et al. Arterial aneurysms associated with cerebral arteriovenous malformations: classification, incidence, and risk of hemorrhage. J Neurosurg 1998;89: 539-46

21. Stefani MA, Porter PJ, terBrugge KG, et al. Large and deep brain arteriovenous malformations are associated with risk of future hemorrhage. Stroke 2002;33:1220-24

22. Illies T, Forkert ND, Saering D, et al. Persistent hemodynamic changes in ruptured brain arteriovenous malformations. Stroke 2012;43:2910-15

23. Raoult H, Ferre JC, Morandi X, et al. Quality-evaluation scheme for cerebral time-resolved 3D contrast-enhanced MR angiography techniques. AJNR Am J Neuroradiol 2010;31:1480-87

24. Wolf RL, Wang J, Detre JA, et al. Arteriovenous shunt visualization in arteriovenous malformations with arterial spin-labeling MR imaging. AJNR Am J Neuroradiol 2008;29:681-87

25. Fiehler J, Illies T, Piening M, et al. Territorial and microvascular perfusion impairment in brain arteriovenous malformations. AJNR Am J Neuroradiol 2009;30:356-61

26. Nett EJ, Johnson KM, Frydrychowicz A, et al. Four-dimensional phase contrast MRI with accelerated dual velocity encoding. J Magn Reson Imaging 2012;35:1462-71

27. Jung B, Honal M, Ullmann P, et al. Highly k-t-space-accelerated phase-contrast MRI. Magn Reson Med 2008;60:1169-77

28. Vakil P, Lee JJ, Mouannes-Srour JJ, et al. Cerebrovascular occlusive disease: quantitative cerebral blood flow using dynamic susceptibility contrast MR imaging correlates with quantitative $\mathrm{H} 2$ [15O] PET. Radiology 2013;266:879-86

29. Mukherjee P, Kang HC, Videen TO, et al. Measurement of cerebral blood flow in chronic carotid occlusive disease: comparison of dynamic susceptibility contrast perfusion MR imaging with positron emission tomography. AJNR Am J Neuroradiol 2003;24: $862-71$ 